\title{
Area and Point Approaches in Fatigue Life Evaluation under Combined Bending and Torsion Loading
}

\author{
A. Karolczuk ${ }^{1, a}$ and E. Macha ${ }^{1, b}$ \\ ${ }^{1}$ Opole University of Technology, Opole, Poland \\ ${ }^{a}$ A.Karolczuk@po.opole.pl, b' E.Macha@po.opole.pl
}

We present a new nonlocal approach to nonuniform stress distribution, consisting in reduction of stresses to representative local ones in the critical plane for fatigue lffe calculation. The shear and normal stresses are averaged in two overlapping areas of different sizes on the critical plane. The proposed method is compared with the point (in critical distance) method and both are verified by fatigue tests under combined bending and torsion. Verification is done for the experimental and calculated fatigue lives with use of two multiaxial fatigue failure criteria.

Keywords: nonlocal approach, critical plane approach, stress gradient.

Introduction. Geometry or loading features of components often causes the formation of nonuniform stress/strain distribution. In case of fatigue loading, cracks are formed under the influence of a stress/strain which undergo changes in time and in some volume of the material. This complicated process must be taken into account in the proper fatigue life estimation.

On the basis of experimental evidences concerning the stress gradient effect on fatigue life, the following phenomena have been revealed: (i) under equal nominal stresses, fatigue lives of specimens subjected to reversed bending are higher than fatigue lives of the same specimens subjected to reversed push-pull loading $[1,2]$; (ii) the initiation of nonpropagating fatigue cracks under loading close to the fatigue limit for defective materials $[3,4]$; (iii) low influence of shear stress gradient on fatigue lives for specimens subjected to reversed torsion $[1,5]$.

The main goal of the present paper is to develop a model of reduction of nonuniform stress distributions around a hot spot in a material to representative local stresses. Representative means taking the above-mentioned (i, ii, iii) phenomena into consideration. The phenomenon (i) allows for the averaging of stresses in some critical space of the material. Fundamental issue is to define the shape and size of this critical space. Definition of these geometrical features is dictated by the other phenomena - (ii) and (iii) The phenomenon (ii) accounts for too small opening stresses active at a small distance from the stress concentrator. Therefore, it is postulated that an averaged value of normal (opening) stresses over the potential crack growth plane, provided that the crack growth must reach the critical value. The phenomenon (iii) is associated only with the macroscopic shear stress gradient which arises, e.g., from torsion of the cylindrical specimens. At the observation scale of a few metallic grains, the macroscopic shear stress gradient is insignificant and has no influence on the crack initiation and especially on the crack growth. However, in the case of a significant shear stress gradient at the mesoscopic scale, which arises, for instance, from notches or defects with sizes of a few metallic grains, it is necessary to take this effect into account during an early crack formation phase. This effect should be considered in the area where crack is formed in the maximum shear stress plane (stage I). The size of this area depends mainly on the material state and loading (type and test conditions) but for most cases this area is very small in comparison to the area where cracks grow on the maximum normal stress plane (stage II).

The paper outlines the model of shear and normal stresses reduction over the critical areas. A new approach is verified using the test results on hour-glass shaped specimens 
subjected to combined cyclic bending and torsion. Moreover, the point approach (the theory of critical distance), which has been analyzed and developed by Taylor and Susmel [6], has been also verified.

Fatigue Tests. Hour-glass shaped specimens made of 18G2A steel (Table 1), with a minimum diameter of $6.5 \mathrm{~mm}$, were subjected to sinusoidal combinations of plane bending and torsion under different moment amplitude ratios $\lambda_{M}=M_{t, a} / M_{b, a}$ (torque/ bending) and two phase shifts $\delta=0$ and $\delta=\pi / 2\left(M_{b}(t)=M_{b, a} \sin (2 \pi f t), M_{t}(t)=\right.$ $\left.M_{t, a} \sin (2 \pi f-\delta), f=20 \mathrm{~Hz}\right)$. Failure of a specimen is defined by about $30 \%$ drop in bending rigidity. The specimens were cut from a 15.8 -mm-diameter drawn bar, machineturned and conventionally polished with progressively finer emery papers.

$\mathrm{T}$ a b 1 e 1

\section{Cyclic Properties of the 18G2A Steel}

\begin{tabular}{|c|c|c|c|c|c|c|c|}
\hline \multicolumn{2}{|c|}{ Torsion: $\tau_{a}=\tau_{a f}\left(N_{\tau} / N_{f}\right)^{1 / m_{\tau}}$} & \multicolumn{2}{c|}{ Push-pull: $\sigma_{a}=\sigma_{a f}\left(N_{\sigma} / N_{f}\right)^{1 / m_{\sigma}}$} & \multicolumn{2}{c|}{$\varepsilon_{a}^{p}=\left(\sigma_{a} / K^{\prime}\right)^{1 / n^{\prime}}$} \\
\hline$\tau_{a f}, \mathrm{MPa}$ & $m_{\tau}$ & $N_{\tau}$, cycles & $\sigma_{a f}, \mathrm{MPa}$ & $m_{\sigma}$ & $N_{\sigma}$, cycles & $K^{\prime}, \mathrm{MPa}$ & $n^{\prime}$ \\
\hline 157 & 9.5 & $1.98 \cdot 10^{6}$ & 204 & 8.2 & $1.24 \cdot 10^{6}$ & 1323 & 0.207 \\
\hline
\end{tabular}

Indices: $a f=$ fatigue limit, $a=$ amplitude, $p=$ plastic, $\tau=$ torsion, $\sigma=$ push-pull, $N=$ cycles.

The Area Method. The proposed area method for reduction of nonuniform distributions of shear and normal stresses to the uniform ones takes into account the phenomena (i), (ii), and (iii) as mentioned above. It is believed that the crack growth or fatigue damage is a local process. It means that the crack initiation period can be described also by the crack growth process. A crack grows from a size of one or a few grains up to the size which defines the component failure. During this process, the crack growth could be governed by different mechanisms (Mode I, Mode II, etc.). Under the conventional uniaxial fatigue tests, the crack grows through the material over the plane which originally had a uniform stress distribution. In the case of fatigue tests with the stress gradient effect, a crack grows over a nonuniformly stressed plane. The local stresses which vary over the potential crack growth plane have an influence on the local crack growth rate and consequently on the total fatigue life. To avoid the complicated process of iterative modeling of the crack growth using the finite element method, it is assumed that the averaged stresses over the potential fracture plane reflect the stress gradient effect. Since the shear crack growing process (stage I) is usually limited to the area of a few grains, the averaging process of shear stresses $\tau_{n s}$ is restricted to that area. The tensile crack growth (stage II) which usually leads to the final failure takes place in the area which defines failure by its size or by the beginning of an unstable crack growth rate. Two components of the stress tensor are distinguished for the averaging process, i.e., shear $\tau_{n s}$ and normal $\sigma_{n}$. The plane orientation whereby these components are determined is defined by multiaxial fatigue failure criterion and is well known as the critical plane. The averaged stresses over two overlapping areas $A_{n s c}$ and $A_{n c}$ are calculated according to the following relations:

$\hat{\tau}_{n s}(t, \vec{n}, \vec{s})=\frac{1}{A_{n s, c}} \int_{A_{n s}} \tau_{n s}(t, \vec{n}, \vec{s}, \varphi, r) d A_{n s}, \quad \hat{\sigma}_{n}(t, \vec{n})=\frac{1}{A_{n, c}} \int_{A_{n}} \sigma_{n}(t, \vec{n}, \varphi, r) d A_{n}$,

where $\vec{n}$ is the normal vector to the critical plane, $\vec{s}$ is the shear vector on the critical plane, $\varphi, r$ are the local coordinates on the critical plane, and $t$ is time. The averaged stresses $\hat{\tau}_{n s}$ and $\hat{\sigma}_{n}$ which are functions of time and the critical plane orientation are 
introduced into the multiaxial fatigue failure criterion. Because the analyzed data pertain to the macroscopic stress gradient, the averaging process over the area of a few grains is not necessary. Only the normal stresses $\sigma_{n}$ are averaged over the area $A_{n, c}$. For simplicity, the shape of the $A_{n . c}$ area is assumed to be semicircular. The failure of the specimens is defined by about $30 \%$ drops in bending rigidity. It means that the averaging process in the cross section of the specimen may be reduced to the semicircular-shaped area of $4.17 \mathrm{~mm}^{2}$ and this value is taken as $A_{n, c}$.

The Point Method. This method assumes that the stressed state, at some critical distance from the hot spot, is responsible for the material failure. If the equivalent stress at this point is high enough, then the crack will propagate and cause the final failure.

Results and Discussion. Stress and strain histories at an arbitrary point $(x, y)$ of the specimen cross section were computed from bending $M_{b}(t)$ and torque $M_{t}(t)$ moments by means of a kinematic hardening model as proposed by Mroz and modified by Garud More details can be found in [7].

Two multiaxial fatigue failure criteria have been verified using test results. One of them is the well known Matake criterion [8],

$$
\hat{\tau}_{n s, a}+\left(2-\frac{\bar{\sigma}_{a f}}{\sigma_{a f}}-1\right) \hat{\sigma}_{n, \max }=\tau_{a f}\left(\frac{N_{\tau}}{N_{\text {calc }}}\right)^{1 / m_{\tau}},
$$

for which the critical plane orientation coincides with the maximum shear stress amplitude. The other criterion is based on two parameters,

$$
N_{\text {calc }}^{o}=N_{o}\left(\frac{\sigma_{a f}}{\hat{\sigma}_{n, \max }}\right)^{m_{v}}, \quad N_{\text {calc }}^{\tau}=N_{\tau}\left(\frac{\tau_{a f}}{\hat{\tau}_{n s, a}}\right)^{m_{\tau}} .
$$

The final fatigue life $N_{\text {calc }}$ is determined by the minimum fatigue life between $N_{\text {calc }}^{\sigma}$ and $N_{\text {calc }}^{\tau} ; \hat{\sigma}_{n, \max }$ is the maximum value in time of the averaged normal stress $\hat{\sigma}_{n}(t)$ on a plane where this stress reaches its maximum, $\hat{\tau}_{n s: a}$ is the amplitude of the averaged shear stress $\hat{\tau}_{n s}(t)$ on a plane where this stress is maximum. Figure 1 gives the results obtained by the proposed area method.
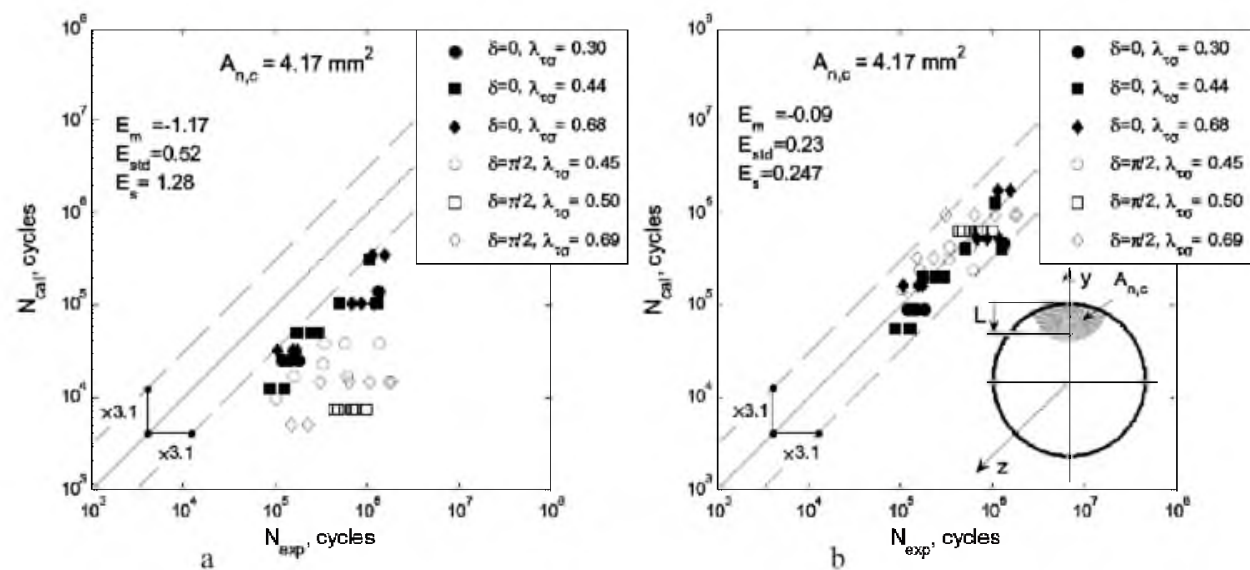

Fig. 1. Comparison of the experimental and calculated fatigue lives: the area method and (a) Matake criterion, (b) two-parameter criterion. [Dashed lines represent the maximum experimental scatter band $\times 3.1$ and $\lambda_{\sigma}=\tau_{x z, a}(L=0) / \sigma_{z z, a}(L=0)$.] 

methods:

The following error parameters were applied for the evaluation of the criteria and

$$
\begin{gathered}
E^{(i)}=\log \frac{N_{\text {calc }}^{(i)}}{N_{\text {exp }}^{(i)}}, \quad E_{m}=\frac{1}{k} \sum_{i=1}^{k} E^{(i)}, \\
E_{\text {std }}=\sqrt{\frac{1}{k-1} \sum_{i=1}^{k}\left(E^{(i)}-E_{m}\right)^{2}}, \quad E_{x}=\sqrt{E_{m}^{2}+E_{s t d}^{2}},
\end{gathered}
$$

where $k$ is a number of specimens $(k=43)$.

Different critical distances $L$ were examined in the case of the point method. The best results used for the Matake criterion were obtained for $L=1.35 \mathrm{~mm}$ (Fig. 2a) and for the two-parameter criterion for $L=0.8 \mathrm{~mm}$ (Fig. 2b).
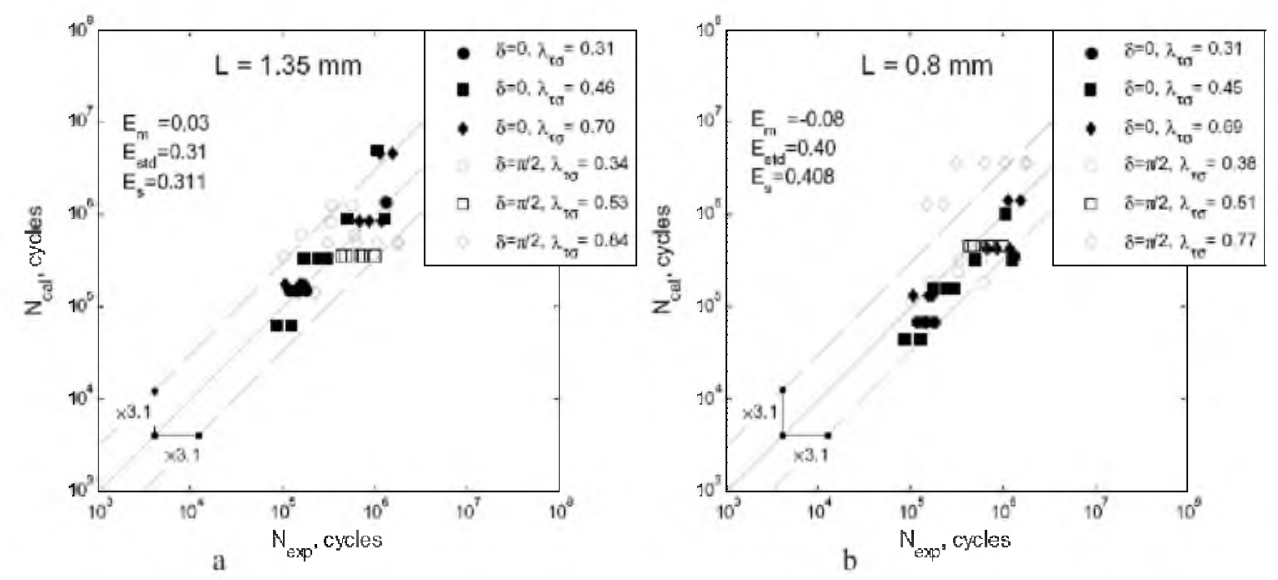

Fig. 2. Comparison of the experimental and calculated fatigue lives: the point method and (a) Matake criterion, (b) two-parameter criterion. [Dashed lines represent the maximum experimental scatter band $\times 3.1$ and $\lambda_{\sigma}=\tau_{x z, a}(L) / \sigma_{z z, a}(L)$.]

Conclusions. Under the investigated test conditions, the experimental and calculated fatigue lives can be successfully correlated with the two-parameter multiaxial fatigue failure criterion based on the critical plane approach and the proposed area method for nonuniform stress reduction. The proposed area method takes into account the different effects of shear and normal stress gradients on the fatigue life. The classical point method neglects this effect, but the results obtained by this method are only a little worse.

1. I. V. Papadopoulos and V. P. Panoskaltsis, Eng. Fract. Mech., 55, No. 4, 513 (1996).

2. F. Morel and T. Palin-Luc, Fatigue Fract. Eng. Mater. Struct., 25, 649 (2002).

3. Y. Murakami and M. Endo, Int. J. Fatigue, 16, 163 (1994).

4. M. Endo and I. Ishimoto, Int. J. Fatigue, 28, 592 (2006).

5. D. McClaflin and A. Fatemi, Int. J. Fatigue, 26, 773 (2004).

6. L. Susmel and D. Taylor, Int. J. Fatigue, 28, 417 (2006).

7. A. Karolczuk, Eng. Fract. Mech., 73, 1629 (2006).

8. T. Matake, Bull. JSME, 20 (141), 257 (1977).

Received 28. 06. 2007 